\title{
Editorial: Spatial Dependency, Spillovers and Farm Efficiency
}

\author{
Francisco J. Areal ${ }^{\text {** }}$ and Valerien O. Pede ${ }^{2}$ \\ ${ }^{1}$ Centre for Rural Economy, Newcastle University, Newcastle upon Tyne, United Kingdom, ${ }^{2}$ International Rice Research \\ Institute (IRRI), Los Baños, Philippines
}

Keywords: stochastic frontier analysis, sustainable farm production, spatial heterogeneity, farm efficiency, farm profitability, land-use management

\section{Editorial on the Research Topic}

\section{Spatial Dependency, Spillovers and Farm Efficiency}

To enhance sustainable agricultural production and land-use governance, a good understanding of the drivers behind farm-level production efficiency is required. Although spatial aspects such as climate, soil, altitude, culture, and infrastructure may well be correlated with farm production, these are rarely accounted for in efficiency analysis. Not accounting for such factors may lead to biased farm-level efficiency estimates. Spatial characteristics, both observed and unobserved by researchers, should be incorporated into efficiency analysis to provide robust advice to multi-level governance. Indeed, not accounting for such spatial heterogeneities may lead to biased results and misleading recommendations to policymakers, extension agents, farmers, and land managers.

The five articles contributing to the Research Topic on spatial dependency, spillovers, and farm efficiency present a variety of approaches and techniques that can be used to account for spatial aspects and incorporate them into the analysis of farm-level production, efficiency, profitability, land-use management, and sustainability. Overall, these articles fulfill the following objectives:

OPEN ACCESS

Edited and Reviewed by:

Ole Mertz,

University of Copenhagen, Denmark

*Correspondence:

Francisco J. Areal

francisco.areal-borrego@ newcastle.ac.uk

Specialty section:

This article was submitted to Land, Livelihoods and Food Security, a section of the journal

Frontiers in Sustainable Food Systems

Received: 08 January 2022

Accepted: 28 January 2022

Published: 18 February 2022

Citation:

Areal FJ and Pede VO (2022) Editorial: Spatial Dependency, Spillovers and Farm Efficiency.

Front. Sustain. Food Syst. 6:850806. doi: 10.3389/fsufs.2022.850806
(1) Contribute to the efficiency literature in identifying different ways to account for unobserved spatial heterogeneity including location and social interaction in efficiency modeling.

(2) Provide practitioners with a set of appropriate tools and approaches to incorporate spatial analysis and/or spillover effects into land use, land-use change, farm production, and efficiency.

(3) Help practitioners provide sound recommendations to stakeholders for better landuse governance.

More specifically, Areal and Pede show the importance of accounting for unobserved spatial heterogeneity within stochastic frontier analysis to provide sound recommendations by comparing standard and spatial stochastic frontier production models that account for unobserved spatial heterogeneity on both the production and efficiency side of the function. Areal and Pede use a sample of 496 irrigated and rainfed rice farms located within $13 \mathrm{~km}$, a relatively close distance, to explain how small networks of relatively close farmers (i.e., spatial dependency associated with information sharing) are associated with rice production and efficiency.

Shrestha et al. also use a stochastic production function approach to focus their analysis on the efficiency, productivity, and profitability of a sample of 325 smallholder vegetable farmers in Nepal representing different agro-ecological areas. The authors highlight the role of increasing farmers' profit efficiency to improve their livelihoods, nutrition security, and income. Shrestha et al. identified the use of improved seed varieties, access to information (in particular output marketing), distance to market, contacting extension agents, and being a female farmer as determinant factors for improving farmers' profit efficiency in vegetable farming in Nepal, leading to a set of policy recommendations. Interestingly, the authors found that farmers' access to credit was negatively associated with profit efficiency. 
Singbo et al. analyse the role of specialization in the economic performance of 239 vegetable farmers in Benin using a Bayesian non-neutral stochastic input distance model, which accounts for the effects of input composition on efficiency. The authors advocate for the use of this approach to account for unobserved sources of production inefficiency. Singbo et al. examine the association between spatial heterogeneity in production efficiency by incorporating information on soil fertility. Farmers' degree of specialization, measured as a normalized Hirschman index of the concentration of output shares for each vegetable crop, was found to be positively associated with technical efficiency in vegetable production. The authors also investigate whether or not economies of scope are present in vegetable farming, finding that they were not present and hence providing low incentives for farmers to specialize.

Mamiit et al. highlight the need to develop novel approaches to achieve sustainable rice production that meets the increase in demand. Mamiit et al. combined the use of a stochastic frontier analysis with spatial hot-spot analysis to identify key geographical areas for policy intervention to increase rice productivity in Central Luzon, Philippines. The authors find that access to irrigation water is key to increasing farm efficiency. Consequently, Mamiit et al. recommend that, when planning policy interventions, dwelling and farm locations need to be taken into account to strategically target areas where production gaps due to insufficient access to water exist.

Vittis et al. investigate the potential for improving farm profitability and sustainable intensification through the use of integrated crop and livestock systems for farms located within less favorable areas (LFAs) in England. By focusing on LFAs, the authors avoid some of the potential production heterogeneity. Vittis et al. use a linear programming model (LPM) with different measures for profitability (annual gross margin and net farm income). The authors find that replacing grassland may be needed to increase profitability of the production systems. They advocate for policies that provide farmers with access to knowledge transfer networks and with a range of structural adoption alternatives that lead to integrated crop and livestock systems in LFAs.
To conclude, we can obtain several key messages from this Research Topic on spatial dependency, spillovers, and farm efficiency. First, one of the main aims of research on farm-level production, efficiency, profitability, land-use management, and sustainability is to inform policymakers and land-use managers. There is a need for research in these areas to acknowledge the limitations associated with not being able to observe all spatial aspects within farm-level production, efficiency, profitability, land-use management, and sustainability analysis as well as the potential consequences of not doing so. Second, we show how such limitations could be curbed by using several approaches that can account for unobserved and observed spatial heterogeneity. Third, social interaction and in particular knowledge transfer networks are important drivers that need to be taken into account in farm efficiency and land-use management analysis. Lastly, in order to provide sound policy recommendations, farm location data represent crucial information to account for any unobserved spatial heterogeneity. This information, combined with other geographic reference datasets (e.g., weather, soil quality), can be incorporated into analyses, thus making this a powerful tool for providing policy-making advice.

\section{AUTHOR CONTRIBUTIONS}

FA and VP have prepared and written this editorial. All authors contributed to the article and approved the submitted version.

Conflict of Interest: The authors declare that the research was conducted in the absence of any commercial or financial relationships that could be construed as a potential conflict of interest.

Publisher's Note: All claims expressed in this article are solely those of the authors and do not necessarily represent those of their affiliated organizations, or those of the publisher, the editors and the reviewers. Any product that may be evaluated in this article, or claim that may be made by its manufacturer, is not guaranteed or endorsed by the publisher.

Copyright (C) 2022 Areal and Pede. This is an open-access article distributed under the terms of the Creative Commons Attribution License (CC BY). The use, distribution or reproduction in other forums is permitted, provided the original author(s) and the copyright owner(s) are credited and that the original publication in this journal is cited, in accordance with accepted academic practice. No use, distribution or reproduction is permitted which does not comply with these terms. 